Research Article

\title{
A Net with Applications for Continuity in a Fuzzy Soft Topological Space
}

\author{
Rui Gao and Jianrong $W u(D)$ \\ School of Mathematics and Physics, Suzhou University of Science and Technology, Suzhou, Jiangsu 215009, China \\ Correspondence should be addressed to Jianrong Wu; jrwu@mail.usts.edu.cn
}

Received 24 March 2020; Revised 22 May 2020; Accepted 1 June 2020; Published 30 June 2020

Academic Editor: Suzanne M. Shontz

Copyright (c) 2020 Rui Gao and Jianrong Wu. This is an open access article distributed under the Creative Commons Attribution License, which permits unrestricted use, distribution, and reproduction in any medium, provided the original work is properly cited.

In this paper, the concept of a fuzzy soft point is redefined, and the definition of a fuzzy soft net in a fuzzy soft topological space is given. On this basis, the convergence of a fuzzy soft net is defined by using the Q-neighborhood theory, and the continuity of fuzzy soft mappings is characterized by the net. The obtained results demonstrate that the concepts proposed in this paper are very suitable and will provide powerful research tools for further research in this field.

\section{Introduction}

In 1965, Zadeh introduced the concept of fuzzy sets in his classic work [1]. In 1999, Molodtsov [2] introduced the theory of soft sets, which have been applied in several fields, including the smoothness of functions, game theory, Riemann integration, and the theory of probability [3]. In 2001, Maji et al. [4] combined fuzzy sets with soft sets and proposed the concept of fuzzy soft sets. Since then, many researchers have applied fuzzy soft sets to group theory [5], decision making, and medical diagnosis [6,7]. In 2009, Kharal and Ahmad [8] studied the properties of fuzzy soft images and fuzzy soft inverse images of fuzzy soft sets.

In 2011, Tanay and Kandemir [9] proposed the concept of a fuzzy soft set in a fuzzy soft topological space and explained some of its structural properties. They also claimed that fuzzy soft topological spaces may be used in the theory of information systems. In 2012, Mahanta and Das [10] introduced the definition of a fuzzy soft point and its neighborhood. They also studied the interior and closure of a fuzzy soft set and investigated the separation axioms and connectedness. Varol and Aygün [11] introduced the fuzzy soft continuity of fuzzy soft mappings. In 2013, Gunduz (Aras) and Bayramov [3] presented fuzzy soft continuous mappings, fuzzy soft open and fuzzy soft closed mappings, and fuzzy soft homeomorphism. In 2014, Ping et al. [12] proposed the sum of fuzzy soft topological spaces. In 2016, Mishra and Srivastava [13] studied compactness in fuzzy soft topological spaces. In 2017, Kandil et al. [14] discussed the connectedness of fuzzy soft sets. Riaz and Hashmi [15] proposed the concept of fuzzifying soft sets, called fuzzy parameterized fuzzy soft sets (FPFS-sets). Mahanta and Das [16] studied fuzzy soft closure and the fuzzy soft interior. In 2018, Abbas et al. [17] explored connectedness in fuzzy soft topological spaces. In 2019, Riaz and Tehrim [18] proved some properties of bipolar fuzzy soft topology (BFS-topology) via the use of the concept of the Q-neighborhood.

In 2012, Roy and Samanta [19] redefined the concept of fuzzy soft topology and obtained some basic results. In a subsequent work [20], they adopted a new definition of a fuzzy soft point, proposed the concepts of quasi-coincidence and Q-neighborhoods, and demonstrated the relationship between the limit point and the closure of a fuzzy soft set.

As pointed out in Example 1 in this paper, the existing concept of fuzzy soft points does not satisfy selectivity, which makes it difficult for a fuzzy soft point to play its expected role. Therefore, a more suitable definition of a fuzzy soft point must be given. Moreover, as is commonly known, the net plays an important role in classical topology theory; however, the concept of the net has not been introduced into fuzzy soft topological spaces. 
In view of these considerations, this paper first redefines the concept of a fuzzy soft point and introduces the net into fuzzy soft topological spaces. The continuity of a mapping in fuzzy soft topological spaces with the use of a net is then studied. The remainder of this article is organized as follows. In Section 2, some necessary concepts of fuzzy soft sets and fuzzy soft topological spaces are recalled. In Section 3, fuzzy soft points are refined and the notion of a fuzzy soft net consisting of fuzzy soft points is introduced. By using the theory of the Q-neighborhood, the concept of the convergence of a fuzzy soft net is introduced. In Section 4, the net is applied to characterize the continuity of fuzzy soft mappings. Section 5 presents a discussion of convergence for a net of fuzzy soft mappings. In Section 6, an example of the application of the fuzzy soft set theory to medical diagnosis is provided. Finally, the conclusion of this paper is given in Section 7.

\section{Preliminaries}

Throughout this paper, $U$ refers to an initial universe and $E$ is the set of all parameters of $U$. In this case, $U$ is also denoted by $(U, E) . I^{U}$ is the set of all fuzzy subsets over $U$, where $I=[0,1]$. The elements $\overline{0}, \overline{1} \in I^{U}$, respectively, refer to the functions $\overline{0}(x)=0$ and $\overline{1}(x)=1$ for all $x \in U$. For an element $A \in I^{U}$, if there exists an $x \in U$ such that $A(x)=\lambda>0$ and $A(y)=0, \forall y \in U \backslash\{x\}$, then $A$ is called a fuzzy point over $U$ and is denoted by $x_{\lambda}$, and $x$ and $\lambda$ are called the support and height of $x_{\lambda}$, respectively. The set of all fuzzy points over $U$ is denoted by $\operatorname{FP}(U)$.

The following definitions in this section were obtained from the existing literature $[19,20]$.

Definition 1. Let $A \subseteq E$. A mapping $F_{A}: E \longrightarrow I^{U}$, defined by $F_{A}(e)=\mu_{F_{A}}^{e}$ (a fuzzy subset of $U$ ), is called a fuzzy soft set over $(U, E)$, where $\mu_{F_{A}}^{e}=\overline{0}$ if $e \in E \backslash A$ and $\mu_{F_{A}}^{e} \neq \overline{0}$ if $e \in A$.

If $A=E$, then $F_{A}$ is shortened to $F$.

The set of all fuzzy soft sets over $(U, E)$ is denoted by $\mathrm{FS}(U, E)$.

The fuzzy soft set $F_{\phi} \in \mathrm{FS}(U, E)$ is called the null fuzzy soft set and is denoted by $\widetilde{\Phi}$. Here, $F_{\phi}(e)=\overline{0}$ for every $e \in E$.

For $F_{E} \in \mathrm{FS}(U, E)$, if $F_{E}(e)=\overline{1}$ for all $e \in E$, then $F_{E}$ is called the absolute fuzzy soft set and is denoted by $\widetilde{E}$.

Let $F_{A}, F_{B} \in \mathrm{FS}(U, E)$. If $F_{A}(e) \subseteq F_{B}(e)$ for all $e \in E$, then $F_{A}$ is said to be a fuzzy soft subset of $F_{B}$ and is denoted by $F_{A} \widetilde{\simeq} F_{B}$ or $F_{B} \supseteq F_{A}$. In addition, $F_{A} \tilde{\nsubseteq} F_{B}$ means that $F_{A}$ is not a fuzzy soft subset of $F_{B}$. If $F_{A} \widetilde{\subseteq} F_{B}$ and $F_{B} \widetilde{\simeq} F_{A}$, then $F_{A}$ and $F_{B}$ are said to be equivalent, which is denoted by $F_{A}=F_{B}$.

Remark 1. If $F_{A} \widetilde{\subseteq} F_{B}$, then $A \subseteq B$.

Definition 2. Let $F_{A}, F_{B} \in \mathrm{FS}(U, E)$.

(1) The complement of $F_{A}$, denoted by $F_{A}^{c}$, is defined as

$$
F_{A}^{c}(e)= \begin{cases}\overline{1}-F_{A}(e), & \text { for } e \in A \\ \overline{1}, & \text { otherwise }\end{cases}
$$

(2) The union of $F_{A}$ and $F_{B}$, denoted by $F_{C}=F_{A} \tilde{\cup} F_{B}$, is defined as $F_{C}(e)=F_{A}(e) \cup F_{B}(e)$ for all $e \in E$, where $C=A \cup B$.

(3) The intersection of $F_{A}$ and $F_{B}$, denoted by $F_{C}=F_{A} \tilde{\cap} F_{B}$, is defined as $F_{C}(e)=F_{A}(e) \cap F_{B}(e)$ for all $e \in E$, where $C=A \cap B$.

Similarly, the union (intersection) of a family of fuzzy soft sets $\left\{F_{C_{\alpha}}: \alpha \in \Lambda\right\}$ can be defined and is denoted by $\tilde{\mathrm{U}}_{\alpha \in \Lambda} F_{C_{\alpha}}\left(\tilde{\mathrm{U}}_{\alpha \in \Lambda} F_{C_{\alpha}}\right)$, where $\Lambda$ is an arbitrary index set.

Remark 2. It is clear that

(1) $\widetilde{\Phi}^{c}=\widetilde{E}, \widetilde{E}^{c}=\widetilde{\Phi}$

(2) $\left(\tilde{\cup}_{\alpha \in \Lambda} F_{A_{\alpha}}\right)^{c}=\tilde{\cap}_{\alpha \in \Lambda} F_{A_{\alpha}}^{c},\left(\tilde{\cap}_{\alpha \in \Lambda} F_{A_{\alpha}}\right)^{c}=\tilde{U}_{\alpha \in \Lambda} F_{A_{\alpha}}^{c}$

Definition 3. A fuzzy soft topology $\tau$ over $(U, E)$ is a family of fuzzy soft sets over $(U, E)$ satisfying the following properties:

(1) $\widetilde{\Phi}, \widetilde{E} \in \tau$

(2) If $F_{A}, F_{B} \in \tau$, then $F_{A} \tilde{\cap} F_{B} \in \tau$

(3) If $F_{A_{\alpha}} \in \tau$ for all $\alpha \in \Lambda$ (any index set), then $\tilde{U}_{\alpha \in \Lambda} F_{A_{\alpha}} \in \tau$

If $\tau$ is a fuzzy soft topology over $(U, E)$, the triple $(U, E, \tau)$ is said to be a fuzzy soft topological space. Each element of $\tau$ is called an open set. If $F_{A}^{c}$ is an open set, then $F_{A}$ is called a closed set.

Definition 4. Let $(U, E, \tau)$ be a fuzzy soft topological space, $F_{A} \in \mathrm{FS}(U, E)$ :

(1) The intersection of all closed sets $F_{B} \supseteq F_{A}$ is called the closure of $F_{A}$ and is denoted by $\bar{F}_{A}$

(2) The union of all open subsets of $F_{A}$ over $(U, E, \tau)$ is called the interior of $F_{A}$ and is denoted by int $F_{A}$

Remark 3. Let $F_{A} \in \mathrm{FS}(U, E)$. It is evident that

(1) $\bar{F}_{A}$ is closed and $\operatorname{int} F_{A}$ is open

(2) $F_{A}$ is closed if and only if $F_{A}=\bar{F}_{A}$

(3) $F_{A}$ is open if and only if $F_{A}=\operatorname{int} F_{A}$

\section{Fuzzy Soft Point and Fuzzy Soft Net}

Roy and Samanta [20] defined a fuzzy soft point $F_{e}$ over $(U, E)$ as a special fuzzy soft set such that $F_{e}(a) \neq \overline{0}$ if $a=e$, and $F_{e}(a)=\overline{0}$ if $a \neq e$. For $F_{A} \in \mathrm{FS}(U, E)$, they stated that a fuzzy soft point $F_{e}$ belongs in $F_{A}$, denoted by $F_{e} \in F_{A}$, if and only if $\left(F_{e}(e)\right)(x) \leq\left(F_{A}(e)\right)(x)$ for all $x \in U$.

Example 1. Let $U=\{x, y\}$ and $E=\{e, f\}$. A fuzzy soft point $F_{e}$ is defined as 


$$
\begin{aligned}
& \left(F_{e}(e)\right)(t)= \begin{cases}\frac{3}{5}, & t=x, \\
\frac{2}{3}, & t=y,\end{cases} \\
& F_{e}(f)=\overline{0} .
\end{aligned}
$$

Also, fuzzy soft sets $F_{A}$ and $F_{B}$ are defined as

$$
\begin{aligned}
& \left(F_{A}(e)\right)(t)= \begin{cases}\frac{3}{5}, & t=x, \\
\frac{1}{3}, & t=y,\end{cases} \\
& \left(F_{A}(f)\right)(t) \equiv \frac{1}{2}, t=x, y, \\
& \left(F_{B}(e)\right)(t)= \begin{cases}\frac{2}{5}, & t=x, \\
\frac{2}{3}, & t=y,\end{cases} \\
& \left(F_{B}(f)\right)(t) \equiv \frac{1}{2} \quad t=x, y .
\end{aligned}
$$

Then,

$$
\begin{aligned}
& \left(\left(F_{A} \widetilde{\cup} F_{B}\right)(e)\right)(t)=\left\{\begin{array}{ll}
\frac{3}{5}, & t=x, \\
\frac{2}{3}, & t=y,
\end{array},\right. \\
& \left(\left(F_{A} \widetilde{\cup} F_{B}\right)(f)\right)(t) \equiv \frac{1}{2}, \quad t=x, y,
\end{aligned}
$$

It is clear that $F_{e} \in F_{A} \tilde{\cup} F_{B}$. However, $F_{e} \notin F_{A}$ and $F_{e} \notin F_{B}$.

This example demonstrates that a fuzzy soft point that belongs in the union of two fuzzy soft sets does not need to belong in one of these two fuzzy soft sets.

To overcome this shortcoming, a fuzzy soft point is redefined as follows.

Definition 5. A mapping $\xi: E \longrightarrow I^{U}$ is called a fuzzy soft point over $(U, E)$ if there is an $e \in E$ such that $\xi(e)=x_{\lambda} \in \mathrm{FP}(U)$, and $\xi(a)=\overline{0}$ when $a \in E \backslash\{e\}$.

In this case, $\xi$ is also denoted by $P_{e}^{x_{\lambda}}$, and $e$ is called its parameter support. The set of all fuzzy soft points over $(U, E)$ is denoted by $\operatorname{FSP}(U, E)$.

It is clear that $F_{e}$ in Example 1 is not a fuzzy soft point in the sense of Definition 5.

Example 2. Let $U=\{x, y\}$ and $E=\{e, f\}$. A mapping $F_{e}: E \longrightarrow I^{U}$ is defined as

$$
\begin{array}{r}
\left(F_{e}(e)\right)(t)= \begin{cases}0.2, & t=x, \\
0, & t=y,\end{cases} \\
F_{e}(f)=\overline{0} .
\end{array}
$$

Then, $F_{e}$ is a fuzzy soft point in the sense of Definition 5 and can be written as $P_{e}^{x_{0.2}}$.

In the remainder of this paper, a fuzzy soft point is always referred to as given by Definition 5 and is called a point for short.

For $P_{e}^{x_{\lambda}}, P_{f}^{y_{\beta}} \in \operatorname{FSP}(U, E)$, if $x \neq y$ or $\lambda \neq \beta$ or $e \neq f$, then it is said that $P_{e}^{x_{\lambda}}$ and $P_{f}^{y_{\beta}}$ are different, which is written as $P_{e}^{x_{\lambda}} \neq P_{f}^{y_{\beta}}$.

Theorem 1. Let $P_{e}^{x_{\lambda}} \in F S P(U, E), F_{A}, F_{B} \in F S(U, E)$, and $F_{A_{\alpha}} \in F S(U, E)(\alpha \in \Lambda)$ :

(1) If $P_{e}^{x_{\lambda}} \in F_{A} \tilde{\cup} F_{B}$, then $P_{e}^{x_{\lambda}} \in F_{A}$ or $P_{e}^{x_{\lambda}} \in F_{B}$

(2) $P_{e}^{x_{\lambda}} \in F_{A_{\alpha}}(\forall \alpha \in \Lambda)$ if and only if $P_{e}^{x_{\lambda}} \in \tilde{\cap}_{\alpha \in \Lambda} F_{A_{\alpha}}$

Proof

(1) Because $P_{e}^{x_{\lambda}} \in F_{A} \tilde{\cup} F_{B}$, then $\lambda \leq \mu_{F_{A}}^{e} \widetilde{\cup} F_{B}(x)$; that is, $\lambda \leq \mu_{F_{A}}^{e}(x) \vee \mu_{F_{B}}^{e}(x)$. Therefore, ${ }_{A} \lambda \leq \mu_{F_{A}}^{e}(x)$ or $\lambda \leq \mu_{F_{B}}^{e}(x)$. Hence, $P_{e}^{x_{\lambda}} \in F_{A}$ or $P_{e}^{x_{\lambda}} \in F_{B}$.

(2) $P_{e}^{x_{\lambda}} \in F_{A_{\alpha}}(\forall \alpha \in \Lambda)$ is equal to $\lambda \leq \mu_{F_{A_{\alpha}}}^{e}(x)(\forall \alpha \in \Lambda)$. Equivalently, $\lambda \leq \Lambda_{\alpha \in \Lambda} \mu_{F_{A_{\alpha}}}^{e}(x)$; that is, $P_{e}^{x_{\lambda}} \in \tilde{\cap}_{\alpha \in \Lambda} F_{A_{\alpha}}$.

However, it is clear that Theorem 1(1) does not hold for the union of infinite fuzzy soft sets. For this reason, the concept of quasi-coincidence is subsequently introduced.

Definition 6. A point $P_{e}^{x_{\lambda}}$ is said to be quasi-coincident with $F_{A} \in \mathrm{FS}(U, E)$, which is denoted by $P_{e}^{x_{\lambda}} \tilde{\in} F_{A}$, if $\lambda+\mu_{F_{A}}^{e}(x)>1$.

That $P_{e}^{x_{\lambda}}$ is not quasi-coincident with $F_{A}$ is denoted by $P_{e}^{x_{\lambda}} \nsubseteq F_{A}$.

Remark 4. In the work by Roy and Samanta [20], a fuzzy soft point $F_{e}$ over $(U, E)$ was said to be quasi-coincident with $F_{A} \in \mathrm{FS}(U, E)$ if $\mu_{F_{e}}(x)+\mu_{F_{A}}^{e}(x)>1$ for some $x \in U$. If $F_{e}$ is a fuzzy soft point in the sense of Definition 5 , then the definition of quasi-coincidence in this paper is equivalent to that in the work by Roy and Samanta [20].

Remark 5. It is evident that $P_{e}^{x_{\lambda}} \tilde{\in} F_{A}$ if and only if $P_{e}^{x_{\lambda}} \notin F_{A}^{c}$. Additionally, if $P_{e}^{x_{\lambda}} \tilde{\in} F_{A}$, then there exists $0<\mu<\lambda$ such that $P_{e}^{x_{1-\mu}} \in F_{A}$.

Theorem 2. Let $F_{A}, F_{B}, F_{A_{\alpha}} \in F S(U, E) \quad(\forall \alpha \in \Lambda)$, and $P_{e}^{x_{\lambda}} \in F S P(U, E)$ :

(1) $F_{A} \widetilde{\subseteq} F_{B}$ if and only if $P_{e}^{x_{\lambda}} \tilde{\in} F_{B}$ for any $P_{e}^{x_{\lambda}} \tilde{\epsilon} F_{A}$

(2) $P_{e}^{x_{\lambda}} \tilde{\in} \tilde{\cap}_{\alpha \in \Lambda} F_{A_{\alpha}}$ implies $P_{e}^{x_{\lambda}} \tilde{\in} F_{A_{\alpha}}(\forall \alpha \in \Lambda)$ $P_{e}^{x_{\lambda}} \tilde{\in} F_{A} \tilde{\cap} F_{B}$ if and only if $P_{e}^{x_{\lambda}} \tilde{\in} F_{A}$ and $P_{e}^{x_{\lambda}} \tilde{\in} F_{B}$

(3) $P_{e}^{x_{\lambda}} \tilde{\in} \tilde{\cup}_{\alpha \in \Lambda} F_{A_{\alpha}}$ if and only if there exists an $\alpha_{0} \in \Lambda$ such that $P_{e}^{x_{\lambda}} \tilde{\in} F_{A_{\alpha_{0}}}$

Proof

(1) The necessity is evident. To prove the sufficiency, it is supposed that $P_{e}^{x_{\lambda}} \tilde{\epsilon} F_{B}$ for any point $P_{e}^{x_{\lambda}} \tilde{\in} F_{A}$. If $F_{A} \widetilde{\simeq} F_{B}$ is not true, then there exist an $e \in E$ and $x \in U$ such that $\mu_{F_{A}}^{e}(x)>\mu_{F_{B}}^{e}(x)$. For $\lambda \in(0,1)$ with $\mu_{F_{A}}^{e}(x)>1-\lambda>\mu_{F_{B}}^{e_{A}^{A}}(x)$, the point $P_{e}^{x_{\lambda}} \widetilde{\in} F_{A}$ and 
$P_{e}^{x_{\lambda}} \widetilde{E} F_{B}$, which contradicts the assumption. Thus, $F_{A} \simeq F_{B}$.

(2) If $P_{e}^{x_{\lambda}} \tilde{\epsilon} \tilde{\cap}_{\alpha \in \Lambda} F_{A_{\alpha}}$, then $\wedge_{\alpha \in \Lambda} \mu_{F_{A_{\alpha}}}^{e}(x)+\lambda>1$. Hence, $\mu_{F_{A_{\alpha}}}^{e}(x)+\lambda>1(\forall \alpha \in \Lambda)$; that is, $P_{e}^{x_{\lambda}} \tilde{\in} F_{A_{\alpha}}(\forall \alpha \in \Lambda)$.

From Definition 6, it is clear that $P_{e}^{x_{\lambda}} \tilde{\in} F_{A} \tilde{\cap} F_{B}$ if and only if $\mu_{F_{A} \tilde{\cap} F_{B}}^{e}(x)+\lambda>1$. Equivalently, $\mu_{F_{A}}^{e}(x) \wedge$ $\mu_{F_{B}}^{e}(x)+\lambda>1$. Thus, $\mu_{F_{A}}^{e}(x)+\lambda>1$ and $\mu_{F_{B}}^{e}(x)+$ $\lambda>1$; that is, $P_{e}^{x_{\lambda}} \tilde{\in} F_{A}$ and $P_{e}^{x_{\lambda}} \tilde{\in} F_{B}$.

(3) From Definition 6, it can be seen that $P_{e}^{x_{\lambda}} \tilde{\in} \tilde{\cup}_{\alpha \in \Lambda} F_{A_{\alpha}}$ if and only if $\vee_{\alpha \in \Lambda} \mu_{F_{A_{\alpha}}}^{e}(x)+\lambda>1$. Equivalently, there exists an $\alpha_{0} \in \Lambda$ such that $\mu_{F_{A_{\alpha_{0}}}}^{e}(x)+\lambda>1$; that is, $P_{e}^{x_{\lambda}} \tilde{\in} F_{A_{\alpha_{0}}}$.

Definition 7. Let $F_{A}, F_{B} \in \mathrm{FS}(U, E)$ and $\xi \in \operatorname{FSP}(U, E)$. If $\xi \widetilde{\epsilon} F_{A} \tilde{\cap} F_{B}$, then it is said that $F_{A}$ and $F_{B}$ are quasi-coincident at $\xi$.

Remark 6. It is clear that $F_{A} \tilde{\cap} F_{B} \neq \widetilde{\Phi}$ if and only if $F_{A}$ and $F_{B}$ are quasi-coincident at a point $\xi \in \operatorname{FSP}(U, E)$.

Definition 8. (see the work by Roy and Samanta [20]). A fuzzy soft set $F_{A}$ is said to be quasi-coincident with $F_{B}$, which is denoted by $F_{A} q F_{B}$, if $\mu_{F_{A}}^{e}(x)+\mu_{F_{B}}^{e}(x)>1$ for some $x \in U$ and $e \in A \cap B$.

That $F_{A}$ is not quasi-coincident with $F_{B}$ is denoted by $F_{A} \bar{q} F_{B}$.

Theorem 3. Let $F_{A}, F_{B} \in F S(U, E)$. If $F_{A} q F_{B}$, then $F_{A} \tilde{\cap} F_{B} \neq \widetilde{\Phi}$.

Proof. Suppose that $F_{A} q F_{B}$; then, there exist an $x \in U$ and $e \in A \cap B$ such that $\mu_{F_{A}}^{e}(x)+\mu_{F_{B}}^{e}(x)>1$. Set $\lambda=$ $\max _{\widetilde{n}}\left\{\mu_{F_{A}}^{e}(\underset{\Phi}{x}), \mu_{F_{B}}^{e}(x)\right\}$. Then, $\quad P_{e}^{x_{\lambda}^{B}} \in F_{A} \tilde{\cap} F_{B}$. Hence, $F_{A} \tilde{\cap} F_{B} \neq \widetilde{\Phi}$.

Remark 7. The converse of Theorem 3 does not hold. Indeed, in Example 1,

$$
\begin{aligned}
\left(\left(F_{A} \tilde{\cap} F_{B}\right)(e)\right)(t) & = \begin{cases}\frac{2}{5}, & t=x, \\
\frac{1}{3}, & t=y,\end{cases} \\
\left(\left(F_{A} \tilde{\cap} F_{B}\right)(f)\right)(t) & \equiv \frac{1}{2}, \\
t & =x, y .
\end{aligned}
$$

Because

$$
\begin{aligned}
& \left(F_{A}(e)\right)(x)+\left(F_{B}(e)\right)(x)=\frac{3}{5}+\frac{2}{5}=1, \\
& \left(F_{A}(f)\right)(x)+\left(F_{B}(f)\right)(x)=\frac{1}{2}+\frac{1}{2}=1, \\
& \left(F_{A}(e)\right)(y)+\left(F_{B}(e)\right)(y)=\frac{1}{3}+\frac{2}{3}=1, \\
& \left(F_{A}(f)\right)(y)+\left(F_{B}(f)\right)(y)=\frac{1}{2}+\frac{1}{2}=1,
\end{aligned}
$$

then $F_{A} \bar{q} F_{B}$. However, $F_{A} \tilde{\cap} F_{B} \neq \widetilde{\Phi}$.

Theorem 4. Let $F_{A}, F_{B} \in F S(U, E)$. If there is a $P_{e}^{x_{\lambda}} \in \operatorname{FSP}(U, E)$ with $\lambda \in[0,1 / 2]$ such that $P_{e}^{x_{\lambda}} \tilde{\in} F_{A} \tilde{\cap} F_{B}$, then $F_{A} q F_{B}$.

Proof. Because $P_{e}^{x_{\lambda}} \tilde{\in} F_{A} \tilde{\cap} F_{B}$, via Theorem 2(2), $\mu_{F_{A}}^{e}(x)+$ $\lambda>1$ and $\mu_{F_{B}}^{e}(x)+\lambda>1$; that is, $\mu_{F_{A}}^{e}(x)+\mu_{F_{B}}^{e}(x)>2-2 \lambda$. From $\lambda \in[0,1 / 2], \quad 1 \leq 2-2 \lambda \leq 2$. Therefore, $\mu_{F_{A}}^{e}(x)+$ $\mu_{F_{B}}^{e}(x)>1$; that is, $F_{A} q F_{B}$.

Definition 9. Let $\xi \in \operatorname{FSP}(U, E)$ and $F_{A}, F_{B} \in \operatorname{FS}(U, E)$ :

(1) $F_{A}$ is said to be a neighborhood of $\xi$ if there exists an $F_{B} \in \tau$ such that $\xi \in F_{B} \widetilde{\simeq} F_{A}$

(2) $F_{A}$ is called a Q-neighborhood of $\xi$ if there exists an $F_{B} \in \tau$ such that $\xi \widetilde{\xi} F_{B} \widetilde{\simeq} F_{A}$

The set of all Q-neighborhoods of $\xi$ is denoted by $\mathcal{U}(\xi)$.

In the remainder of this paper, $\Delta$ is a directed set with the partial order “ $\prec$."

Remark 8. It is clear that $\mathcal{U}(\xi)$ is a directed set with the partial order " $\widetilde{\simeq}$."

Definition 10. The mapping $S: \Delta \longrightarrow \operatorname{FSP}(U, E)$ is called a fuzzy soft net in $(U, E)$ and is denoted by $\{S(\delta), \delta \in \Delta\}$, or $S$ for simplicity.

In particular, if there exists an $F_{A} \in \mathrm{FS}(U, E)$ such that $S(\delta) \in F_{A}$ for any $\delta \in \Delta$, then $S$ is said to be a fuzzy soft net in $F_{A}$, or a net for simplicity.

Definition 11. Let $F_{A} \in \mathrm{FS}(U, E)$ and $S=\{S(\delta), \delta \in \Delta\}$ be a net in $(U, E)$. If there exists a $\delta_{0} \in \Delta$ such that $S(\delta) \tilde{\epsilon} F_{A}$ whenever $\delta_{0} \prec \delta$, then $S$ is said to be eventually quasi-coincident with $F_{A}$. If for each $\delta \in \Delta$ there exists a $\delta_{0} \in \Delta$ with $\delta<\delta_{0}$ such that $S\left(\delta_{0}\right) \tilde{\epsilon} F_{A}$, then $S$ is said to be frequently quasi-coincident with $F_{A}$.

Definition 12. A net $\{S(\delta), \delta \in \Delta\}$ in $(U, E, \tau)$ is said to be convergent to a point $\xi$ if $S$ is eventually quasi-coincident with each Q-neighborhood of $\xi$. In this case, $\xi$ is called the limit of $S$ and is denoted by $\lim S(\delta)$.

\section{Fuzzy Soft Continuous Mapping}

In this section, the definition of fuzzy soft continuous mapping is first recalled, and the net is then applied to characterize the continuity.

Definition 13 (see the work of Aygünoğlu and Aygün [5]). Let FS $\left(U_{1}, E_{1}\right)$ and $\mathrm{FS}\left(U_{2}, E_{2}\right)$ be the families of all fuzzy soft sets over $U_{1}$ and $U_{2}$, respectively. Let $\varphi: U_{1} \longrightarrow U_{2}$ and $\psi: E_{1} \longrightarrow E_{2}$ be two functions. Then, the pair $(\varphi, \psi)$ is called a fuzzy soft mapping from $U_{1}$ to $U_{2}$ and is denoted by $(\varphi, \psi)$ : $\mathrm{FS}\left(U_{1}, E_{1}\right) \longrightarrow \mathrm{FS}\left(U_{2}, E_{2}\right):$

(1) Let $F_{A} \in \mathrm{FS}\left(U_{1}, E_{1}\right)$. Then, the image of $F_{A}$ under the fuzzy soft mapping $(\varphi, \psi)$ is the fuzzy soft set over $U_{2}$ defined by $(\varphi, \psi)\left(F_{A}\right)$, where $(\varphi, \psi)\left(F_{A}\right)(k)(y)$ 


$$
=\left\{\begin{array}{ll}
\underset{\varphi(x)=y}{\bigvee} \underset{\psi(e)=k}{\bigvee} F_{A}(e)(x), & \text { if } x \in \varphi^{-1}(y), \\
\overline{0}, & \text { otherwise, }
\end{array} \quad \forall k \in \psi\left(E_{1}\right), \forall y \in U_{2} .\right.
$$

(2) Let $F_{B} \in \mathrm{FS}\left(U_{2}, E_{2}\right)$. Then, the pre-image of $F_{B}$ under the fuzzy soft mapping $(\varphi, \psi)$ is the fuzzy soft set over $U_{1}$ defined by $(\varphi, \psi)^{-1}\left(F_{B}\right)$, where

$$
\begin{aligned}
& (\varphi, \psi)^{-1}\left(F_{B}\right)(e)(x)=F_{B}(\psi(e))(\varphi(x)), \\
& \forall e \in \psi^{-1}\left(E_{2}\right) \forall x \in U_{1} .
\end{aligned}
$$

If both $\varphi$ and $\psi$ are injective (surjective), then the fuzzy soft mapping $(\varphi, \psi)$ is said to be injective (surjective).

The composition of two fuzzy soft mappings $(\varphi, \psi)$ from $\left(U_{1}, E_{1}\right)$ to $\left(U_{2}, E_{2}\right)$ and $(\varphi \prime, \psi \prime)$ from $\left(U_{2}, E_{2}\right)$ to $\left(U_{3}, E_{3}\right)$ is defined as $(\varphi / \circ \varphi, \psi / \circ \psi)$ from $\left(U_{1}, E_{1}\right)$ to $\left(U_{3}, E_{3}\right)$.

Lemma 1 (see the work of Kharal and Ahmad [8]). Let $F_{A}$, $\left(F_{A}\right)_{i} \in F S\left(U_{1}, E_{1}\right)$, and $F_{B},\left(F_{B}\right)_{i} \in F S\left(U_{2}, E_{2}\right), i=1,2$. The following is then obtained:

(1) If $\left(F_{A}\right)_{1} \widetilde{\simeq}\left(F_{A}\right)_{2}$, then $(\varphi, \psi)\left(F_{A}\right)_{1} \tilde{\subseteq}(\varphi, \psi)\left(F_{A}\right)_{2}$

(2) If $\left(F_{B}\right)_{1} \tilde{\subseteq}\left(F_{B}\right)_{2}$, then $(\varphi, \psi)^{-1}\left(F_{B}\right)_{1} \tilde{\subseteq}(\varphi, \psi)^{-1}\left(F_{B}\right)_{2}$

(3) $F_{A} \widetilde{\simeq}(\varphi, \psi)^{-1}\left((\varphi, \psi)\left(F_{A}\right)\right)$; the equality holds if $(\varphi, \psi)$ is injective

(4) $(\varphi, \psi)\left((\varphi, \psi)^{-1}\left(F_{B}\right)\right) \widetilde{\simeq} F_{B}$; the equality holds if $(\varphi, \psi)$ is surjective

It is simple to verify the following lemma.

Lemma 2. Let $(\varphi, \psi)$ be a fuzzy soft mapping, $P_{e}^{x_{\lambda}} \in F S P\left(U_{1}, E_{1}\right)$ and $F_{A} \in F S\left(U_{1}, E_{1}\right)$; then

(1) $(\varphi, \psi)\left(P_{e}^{x_{\lambda}}\right)=P_{\psi(e)}^{\varphi(x)_{\lambda}}$

(2) If $P_{e}^{x_{\lambda}} \tilde{\epsilon} F_{A}$, then $(\varphi, \psi)\left(P_{e}^{x_{\lambda}}\right) \tilde{\epsilon}(\varphi, \psi)\left(F_{A}\right)$

(3) If $P_{e}^{x_{\lambda}} \in F_{A}$, then $(\varphi, \psi)\left(P_{e}^{x_{\lambda}}\right) \in(\varphi, \psi)\left(F_{A}\right)$

Theorem 5. Let $F_{A}, \quad F_{B} \in F S\left(U_{1}, E_{1}\right)$, and $(\varphi, \psi)$ : $\left(U_{1}, E_{1}\right) \longrightarrow\left(U_{2}, E_{2}\right)$ be a fuzzy soft mapping. If $F_{A} q F_{B}$, then $(\varphi, \psi)\left(F_{A}\right) q(\varphi, \psi)\left(F_{B}\right)$.

Proof. Suppose that $F_{A} q F_{B}$. Then, there are an $x \in U_{1}$ and $e \in E_{1}$ such that $F_{A}(e)(x)+F_{B}(e)(x)>1$. Let $F_{A}(e)(x)=\lambda$. Then, $P_{e}^{x_{\lambda}} \in F_{A}$ and $P_{e}^{x_{\lambda}} \tilde{\in} F_{B}$. From Lemma 2 , it can be found that $(\varphi, \psi)\left(P_{e}^{x_{\lambda}}\right) \in(\varphi, \psi)\left(F_{A}\right), \quad(\varphi, \psi)\left(P_{e}^{x_{\lambda}}\right) \tilde{\epsilon}(\varphi, \psi)\left(F_{B}\right)$. Thus,

$$
\begin{array}{r}
\left((\varphi, \psi)\left(F_{B}\right)\right)(\psi(e))(\varphi(x))+\lambda>1, \\
\left((\varphi, \psi)\left(F_{A}\right)\right)(\psi(e))(\varphi(x)) \geq \lambda .
\end{array}
$$

Therefore,

$\left((\varphi, \psi)\left(F_{B}\right)\right)(\psi(e))(\varphi(x))+\left((\varphi, \psi)\left(F_{A}\right)\right)(\psi(e))(\varphi(x))>1$.

Equivalently,

$$
\mu_{(\varphi, \psi)\left(F_{B}\right)}^{\psi(e)}(\varphi(x))+\mu_{(\varphi, \psi)\left(F_{A}\right)}^{\psi(e)}(\varphi(x))>1 .
$$

which implies that $(\varphi, \psi)\left(F_{A}\right) q(\varphi, \psi)\left(F_{B}\right)$.

Definition 14 (see the work by Varol and Aygün [11]). Let $\left(U_{1}, E_{1}, \tau_{1}\right)$ and $\left(U_{2}, E_{2}, \tau_{2}\right)$ be two fuzzy soft topological spaces. A fuzzy soft mapping $(\varphi, \psi):\left(U_{1}, E_{1}, \tau_{1}\right) \longrightarrow$ $\left(U_{2}, E_{2}, \tau_{2}\right)$ is said to be fuzzy soft continuous if $(\varphi, \psi)^{-1}\left(F_{B}\right) \in \tau_{1}, \forall F_{B} \in \tau_{2}$.

If both $(\varphi, \psi):\left(U_{1}, E_{1}, \tau_{1}\right) \longrightarrow\left(U_{2}, E_{2}, \tau_{2}\right)$ and $\left(\varphi^{\prime}, \psi^{\prime}\right)$ : $\left(U_{2}, E_{2}, \tau_{2}\right) \longrightarrow\left(U_{3}, E_{3}, \tau_{3}\right)$ are fuzzy soft continuous, then it is clear that $\left(\varphi^{\prime}, \psi^{\prime}\right) \circ(\varphi, \psi)$ is also fuzzy soft continuous. In fact, for a fuzzy soft set $F_{A}$ on $U_{3}$,

$$
\begin{aligned}
\left(\left(\varphi^{\prime}\right.\right. & \left.\left., \psi^{\prime}\right) \circ(\varphi, \psi)\right)^{-1}\left(F_{A}\right)(e) \\
& =\left(\left(\varphi^{\prime} \circ \varphi, \psi^{\prime} \circ \psi\right)\right)^{-1}\left(F_{A}\right)(e) \\
& =\left(\varphi^{\prime} \circ \varphi\right)^{-1}\left(F_{A}\left(\psi^{\prime}(\psi(e))\right)\right) \\
& =\varphi^{-1}\left(\left(\varphi^{\prime}\right)^{-1}\left(F_{A}\left(\psi^{\prime}(\psi(e))\right)\right)\right) \\
& =(\varphi, \psi)^{-1}\left(\left(\varphi^{\prime}, \psi^{\prime}\right)^{-1}\left(F_{A}\right)\right)(e) .
\end{aligned}
$$

Hence,

$$
\begin{aligned}
& \left(\left(\varphi^{\prime}, \psi^{\prime}\right) \circ(\varphi, \psi)\right)^{-1}\left(F_{A}\right) \\
& =(\varphi, \psi)^{-1}\left(\left(\varphi^{\prime}, \psi^{\prime}\right)^{-1}\left(F_{A}\right)\right) .
\end{aligned}
$$

Theorem 6. Let $\left(U_{1}, E_{1}, \tau_{1}\right)$ and $\left(U_{2}, E_{2}, \tau_{2}\right)$ be two fuzzy soft topological spaces and $(\varphi, \psi):\left(U_{1}, E_{1}, \tau_{1}\right) \longrightarrow$ $\left(U_{2}, E_{2}, \tau_{2}\right)$ be a fuzzy soft mapping. The following are then equivalent:

(1) $(\varphi, \psi):\left(U_{1}, E_{1}, \tau_{1}\right) \longrightarrow\left(U_{2}, E_{2}, \tau_{2}\right)$ is fuzzy soft continuous

(2) For each $\xi \in F S P\left(U_{1}, E_{1}\right)$ and each neighborhood $F_{B}$ of $(\varphi, \psi)(\xi)$, there exists a neighborhood $F_{A}$ of $\xi$ such that $(\varphi, \psi)\left(F_{A}\right) \tilde{\subseteq} F_{B}$

(3) For each $P_{e}^{x_{\lambda}} \in \operatorname{FSP}\left(U_{1}, E_{1}\right)$ and each Q-neighborhood $F_{B}$ of $(\varphi, \psi)\left(P_{e}^{x_{\lambda}}\right)$, there exists a Q-neighborhood $F_{A}$ of $P_{e}^{x_{\lambda}}$ such that $(\varphi, \psi)\left(F_{A}\right) \widetilde{\subseteq} F_{B}$

(4) For each net $S=\{S(\delta), \delta \in \Delta\}$ in $\left(U_{1}, E_{1}\right)$, if $S$ converges to $P_{e}^{x_{\lambda}} \in F S P\left(U_{1}, E_{1}\right)$, then $(\varphi, \psi) \circ S=\{(\varphi, \psi)(S(\delta)), \delta \in \Delta\}$ is a net in $\left(U_{2}, E_{2}\right)$ and converges to $(\varphi, \psi)\left(P_{e}^{x_{\lambda}}\right)$

Proof. $\quad(1) \Rightarrow(2)$ : Suppose that $\xi \in \operatorname{FSP}\left(U_{1}, E_{1}\right)$ and $F_{B}$ is a neighborhood of $(\varphi, \psi)(\xi)$. Then, there exists an $F_{C} \in \tau_{2}$ such that $(\varphi, \psi)(\xi) \in F_{C} \tilde{\subseteq} F_{B}$. Because $(\varphi, \psi)$ is fuzzy soft continuous, $F_{A}=$ $(\varphi, \psi)^{-1}\left(F_{C}\right) \in \tau_{1}$. Then, $F_{A}$ is a neighborhood of $\xi$ and $(\varphi, \psi)\left(F_{A}\right) \widetilde{\subseteq} F_{C} \widetilde{\subseteq} F_{B}$ follows from Lemma 1(4).

(2) $\Rightarrow$ (3): Suppose that $P_{e}^{x_{\lambda}} \in \operatorname{FSP}\left(U_{1}, E_{1}\right)$ and $F_{B}$ is a Q-neighborhood of $(\varphi, \psi)\left(P_{e}^{x_{\lambda}}\right)$. Then, there is an open fuzzy soft set $F_{B^{\prime}} \in \mathscr{U}\left((\varphi, \psi)\left(P_{e}^{x_{\lambda}}\right)\right)$ such that $F_{B^{\prime}} \widetilde{\tilde{\subseteq}} F_{B}$. From Remark 5 and Lemma 2(1), it can be determined 
that there exists $0<\mu<\lambda$ such that $(\varphi, \psi)\left(P_{e}^{x_{1-\mu}}\right) \in F_{B^{\prime}}$. Thus, $F_{B^{\prime}}$ is a neighborhood of $(\varphi, \psi)\left(P_{e}^{x_{1-\mu}}\right)$. Under condition (2), there exists an open neighborhood $F_{A}$ of $P_{e}^{x_{1-\mu}}$ such that $(\varphi, \psi)\left(F_{A}\right) \widetilde{\widetilde{\subseteq}} F_{B^{\prime}}$. Because $0<\mu<\lambda$, $\mu_{F_{A}}^{e}(x) \geq 1-\mu>1-\lambda$. Therefore, $F_{A}$ is a Q-neighborhood of $P_{e}^{x_{\lambda}}$, and $(\varphi, \psi)\left(F_{A}\right) \widetilde{\subseteq} F_{B}$.

(3) $\Rightarrow$ (4): Suppose a net $S=\{S(\delta), \delta \in \Delta\}$ in $\left(U_{1}, E_{1}\right)$ converges to $P_{e}^{\chi_{\lambda}} \in \operatorname{FSP}\left(U_{1}, E_{1}\right)$. For any $F_{B} \in \mathscr{U}$ $\left((\varphi, \psi)\left(P_{e}^{x_{\lambda}}\right)\right)$, under condition (3), there exists an $F_{A} \in \mathscr{U}\left(P_{e}^{x_{\lambda}}\right)$ such that $(\varphi, \psi)\left(F_{A}\right) \tilde{\subseteq} F_{B}$. From the supposition, there is a $\delta_{0} \in \Delta$ such that $S(\delta) \tilde{\epsilon} F_{A}$ whenever $\delta_{0}<\delta$. Lemma 2 implies that $(\varphi, \psi)$ $(S(\delta)) \tilde{\epsilon}(\varphi, \psi)\left(F_{A}\right) \tilde{\subseteq} F_{B} . \quad$ Therefore, $\quad(\varphi, \psi) \circ S=$ $\{(\varphi, \psi)(S(\delta)), \delta \in \Delta\}$ converges to $(\varphi, \psi)\left(P_{e}^{x_{\lambda}}\right)$.

$(4) \Rightarrow(1)$ : This is proven by contradiction. If $(\varphi, \psi)$ is not fuzzy soft continuous, then there is an $F_{B} \in \tau_{2}$ such that $(\varphi, \psi)^{-1}\left(F_{B}\right)$ is not open. From Remark 3(3), it can be found that there exists a $P_{e}^{x_{\lambda}} \in \operatorname{FSP}\left(U_{1}, E_{1}\right)$ such that $P_{e}^{x_{\lambda}} \tilde{\epsilon}(\varphi, \psi)^{-1}\left(F_{B}\right), P_{e}^{x_{\lambda}} \tilde{\notin} \operatorname{int}\left((\varphi, \psi)^{-1}\left(F_{B}\right)\right)$. By Definition $4(2)$, there is an $F_{A} \nsubseteq(\varphi, \psi)^{-1}\left(F_{B}\right)$ for any $F_{A} \in \mathcal{U}\left(P_{e}^{x_{\lambda}}\right)$. It follows from Theorem 2(1) that there exists a $\xi\left(F_{A}\right) \in \operatorname{FSP}\left(U_{1}, E_{1}\right)$ such that $\xi\left(F_{A}\right) \tilde{\epsilon} F_{A}$ and $\xi\left(F_{A}\right) \widetilde{\notin}(\varphi, \psi)^{-1}\left(F_{B}\right)$. Recall from Remark 8 that $\mathcal{U}\left(P_{e}^{x_{\lambda}}\right)$ is a directed set with the partial order " $\widetilde{\subseteq}$ "; thus, a net $S=\left\{\xi\left(F_{A}\right): F_{A} \in \mathcal{U}\left(P_{e}^{x_{\lambda}}\right)\right\}$ in $\left(U_{1}, E_{1}\right)$ is obtained. It is easy to verify that $S$ converges to $P_{e}^{x_{\lambda}}$. Under condition (4), the net $(\varphi, \psi) \circ S$ converges to $(\varphi, \psi)\left(P_{e}^{x_{\lambda}}\right)$. Recall that $P_{e}^{x_{\lambda}} \tilde{\epsilon}(\varphi, \psi)^{-1}\left(F_{B}\right)$, or $(\varphi, \psi)$ $\left(P_{e}^{x_{\lambda}}\right) \tilde{\epsilon} F_{B} \in \tau_{2}$; it is known that $F_{B}$ is a Q-neighborhood of $(\varphi, \psi)\left(P_{e}^{x_{\lambda}}\right)$. Thus, $(\varphi, \psi) \circ S$ is eventually quasicoincident with $F_{B}$, which conflicts with the fact that $\xi\left(F_{A}\right) \widetilde{\notin}(\varphi, \psi)^{-1}\left(F_{B}\right)$, or $(\varphi, \psi)\left(\xi\left(F_{A}\right)\right) \widetilde{\notin} F_{B}$, for any $F_{A} \in \mathscr{U}\left(P_{e}^{x_{\lambda}}\right)$. Therefore, $(\varphi, \psi)$ is fuzzy soft continuous.

\section{Convergence for a Net of Fuzzy Soft Mappings}

Let $\left(U_{1}, E_{1}, \tau_{1}\right)$ and $\left(U_{2}, E_{2}, \tau_{2}\right)$ be two fuzzy soft topological spaces. $\operatorname{FSM}\left(U_{1}, U_{2}\right)$ denotes the set of all mappings of $\left(U_{1}, E_{1}, \tau_{1}\right)$ into $\left(U_{2}, E_{2}, \tau_{2}\right)$.

Definition 15. A net $\left\{(\varphi, \psi)_{\mu}, \mu \in M\right\}$ in $\operatorname{FSM}\left(U_{1}, U_{2}\right)$ is said to be convergent to $(\varphi, \psi) \in \operatorname{FSM}\left(U_{1}, U_{2}\right)$ if, for every point $\xi \in \operatorname{FSP}\left(U_{1}, E_{1}\right)$ and for every $F_{B} \in \mathscr{U}((\varphi, \psi)(\xi))$, there exist a $\mu_{0} \in M$ and $F_{A} \in \mathcal{U}(\xi)$ such that $(\varphi, \psi)_{\mu}\left(F_{A}\right) \tilde{\subseteq} F_{B}$ for every $\mu \in M$ with $\mu \geq \mu_{0}$.

Theorem 7. A net $\left\{(\varphi, \psi)_{\mu}, \mu \in M\right\}$ in $\operatorname{FSM}\left(U_{1}, U_{2}\right)$ converges to $(\varphi, \psi) \in F S M\left(U_{1}, U_{2}\right)$ if and only if, for every net $\{S(\delta), \delta \in \Delta\}$ in $\left(U_{1}, E_{1}, \tau_{1}\right)$ which converges to a point $\xi \in F S M\left(U_{1}, E_{1}\right)$, the net $\left\{(\varphi, \psi)_{\mu}(S(\delta)),(\delta, \mu) \in \Delta \times M\right\}$ converges to the point $(\varphi, \psi)(\xi) \in F S P\left(U_{2}, E_{2}\right)$, where $\Delta \times M$ is the product of $\Delta$ and $M$.

Proof. (Necessity) Let $\{S(\delta), \delta \in \Delta\}$ be a net in $\operatorname{FSM}\left(U_{1}, U_{2}\right)$ that converges to $\xi \in \operatorname{FSP}\left(U_{1}, E_{1}\right)$ and let $F_{B} \in \mathscr{U}((\varphi, \psi)(\xi))$ arbitrarily. By assumption, there exist an $F_{A} \in \mathscr{U}(\xi)$ and $\mu_{0} \in M$ such that $(\varphi, \psi)_{\mu}\left(F_{A}\right) \tilde{\subseteq} F_{B}$ for any $\mu \in M$ with $\mu_{0}<\mu$. Because $\{S(\delta), \delta \in \Delta\}$ converges to $\xi$, there is a $\delta_{0} \in \Delta$ such that $S(\delta) \tilde{\epsilon} F_{A}$ for every $\delta \in \Delta$ with $\delta_{0}<\delta$. Then, for every $(\delta, \mu) \in \Delta \times M$ with $\left(\delta_{0}, \mu_{0}\right)<(\delta, \mu)$, it follows from Lemma $2(2)$ that $(\varphi, \psi)_{\mu}(S(\delta)) \tilde{\epsilon}(\varphi, \psi)_{\mu}\left(F_{A}\right) \tilde{\subseteq} F_{B}$, which means $\left\{(\varphi, \psi)_{\mu}, \mu \in M\right\}$ converges to $(\varphi, \psi)$.

(Sufficiency) Proceeding by contradiction, it is supposed that there are a $\xi \in \operatorname{FSP}\left(U_{1}, E_{1}\right), F_{B} \in \mathcal{U}((\varphi, \psi)(\xi))$ such that, for every $\mu \in M$ and for every $F_{A} \in \mathcal{U}(\xi)$, there exists a $\mu \prime \geq \mu$ such that $(\varphi, \psi)_{\mu^{\prime}}\left(F_{A}\right) \nsubseteq F_{B}$. From Theorem 2(1) and Lemma 2(1), a point $\xi\left(F_{A}\right) \in \operatorname{FSP}\left(U_{1}, E_{1}\right)$ is obtained such that $\xi\left(F_{A}\right) \tilde{\epsilon} F_{A}$ and $(\varphi, \psi)_{\mu^{\prime}}\left(\xi\left(F_{A}\right)\right) \widetilde{\notin} F_{B}$. As can be determined from Remark 8, the net $S=\left\{\xi\left(F_{A}\right): F_{A} \in \mathcal{U}(\xi)\right\}$ converges to $\xi$. However, the net $\left\{(\varphi, \psi)_{\mu}\left(S\left(F_{A}\right)\right),\left(F_{A}, \mu\right) \in \mathcal{U}(\xi) \times M\right\}$ does not converge to $(\varphi, \psi)(\xi)$. This conflicts with the condition that $\left\{(\varphi, \psi)_{\mu}, \mu \in M\right\}$ converges to $(\varphi, \psi)$.

\section{Application of Fuzzy Soft Set Theory to Medical Diagnosis}

In a hospital, some doctors usually decide what disease a patient is suffering from by observing the patient's symptoms. However, due to the complexity of symptoms, it is difficult to find the precise relationship between diseases and symptoms. The concept of fuzzy soft sets partially resolves this difficulty.

Suppose that the initial universe $U=\left\{x_{1}, x_{2}, \ldots, x_{m}\right\}$ is the set of all the disease objects that the patient may be infected with, and the set of parameters $E=\left\{e_{1}, e_{2}, \ldots, e_{n}\right\}$ is all of the patient's symptoms. Generally speaking, from a symptom $e \in E$, one cannot completely determine the corresponding disease $x \in U$; however, one can determine the membership degree in which object $x \in U$ holds parameter $e \in E$, which is denoted by $\mu_{F}^{e}(x)$; that is, for every $e \in E$, there is a fuzzy subset $F(e)$ of $U$. Obviously, the mapping $F: E \longrightarrow I^{U}$ is a fuzzy soft set over $(U, E)$.

Let

$$
\mu\left(x_{i}\right)=\sum_{k=1}^{n} \mu_{F}^{e_{k}}\left(x_{i}\right)
$$

If $\mu\left(x_{i_{0}}\right)=\max _{1 \leq i \leq m} \mu\left(x_{i}\right)$, then it may be claimed that the patient has disease $x_{i_{0}}$.

\section{Conclusions}

In this paper, the new concepts of fuzzy soft points and fuzzy soft nets were introduced to fuzzy soft topological spaces. On these bases, the fuzzy soft net was used to accurately describe the convergence, which was used to characterize the continuity. Moreover, the convergence for a net of fuzzy soft mappings was investigated. The obtained results demonstrate that the concepts proposed in this paper are very useful and will provide powerful research tools for further research in this field. Particularly, the convergence of fuzzy soft nets may be used to characterize some important properties of fuzzy soft topological spaces, such as closure, separation, compactness, etc. 


\section{Data Availability}

No data were used to support this study.

\section{Conflicts of Interest}

The authors declare that they have no conflicts of interest.

\section{Acknowledgments}

This work was supported by the National Natural Science Foundation of China (11971343) and the Postgraduate Research \& Practice Innovation Program of Jiangsu Province (KYCX19_2015).

\section{References}

[1] L. A. Zadeh, "Fuzzy sets," Information and Control, vol. 8, no. 3, pp. 338-353, 1965.

[2] D. Molodtsov, "Soft set theory-first results," Computers and Mathematics with Applications, vol. 37, no. 4-5, pp. 19-31, 1999.

[3] C. Gunduz (Aras) and S. Bayramov, "Some results on fuzzy soft topological spaces," Mathematical Problems in Engineering, vol. 2013, Article ID 835308, 10 pages, 2013.

[4] P. K. Maji, R. Biswas, and A. R. Roy, "Fuzzy soft sets," Journal of Fuzzy Mathematics, vol. 9, no. 3, pp. 589-602, 2001.

[5] A. Aygünoğlu and H. Aygün, "Introduction to fuzzy soft groups," Computers and Mathematics with Applications, vol. 58, no. 6, pp. 1279-1286, 2009.

[6] Z. Kong, L. Gao, and L. Wang, "A fuzzy soft set theoretic approach to decision making problems," Journal of Computational and Applied Mathematics, vol. 223, no. 2, pp. 540542,2009

[7] S. Alkhazaleh, A. R. Salleh, and N. Hassan, "Fuzzy parameterized interval-valued fuzzy soft set," Applied Mathematical Sciences, vol. 5, pp. 3335-3346, 2011.

[8] A. Kharal and B. Ahmad, "Mappings on fuzzy soft classes," Advances in Fuzzy Systems, vol. 2009, Article ID 407890, 6 pages, 2009.

[9] B. Tanay and M. B. Kandemir, "Topological structure of fuzzy soft sets," Computers \& Mathematics with Applications, vol. 61, no. 10, pp. 2952-2957, 2011.

[10] J. Mahanta and P. K. Das, "Results on fuzzy soft topological spaces," 2012, https://arxiv.org/abs/1203.0634.

[11] B. P. Varol and H. Aygün, "Fuzzy soft topology," Hacettepe Journal of Mathematics and Statistics, vol. 41, no. 3, pp. 407-419, 2012.

[12] J. S. Ping, T. Wu, and C. Z. Yang, "Sum spaces in fuzzy soft topological spaces," Fuzzy Systems and Mathematics, vol. 28, no. 4, pp. 69-73, 2014.

[13] S. Mishra and R. Srivastava, "Fuzzy soft compact topological spaces," Journal of Mathematics, vol. 2016, Article ID 2480842, 7 pages, 2016.

[14] A. Kandil, O. A. El-Tantawy, S. A. El-Sheikh, and S. S. S. El-Sayed, "Fuzzy soft connected sets in fuzzy soft topological spaces II," Journal of the Egyptian Mathematical Society, vol. 25, no. 2, pp. 171-177, 2017.

[15] M. Riza and M. R. Hashmi, "Fuzzy parameterized fuzzy soft topology with applications," Annals of Fuzzy Mathematics and Informatics, vol. 13, no. 5, pp. 593-613, 2017.

[16] J. Mahanta and P. K. Das, "Fuzzy soft topological spaces," Journal of Intelligent \& Fuzzy Systems, vol. 32, no. 1, pp. 443-450, 2017.
[17] S. E. Abbas, El-sayed El-sanowsy, and A. Atef, "Connectedness in fuzzy soft topological spaces," Bulletin of the Brazilian Mathematical Society, New Series, vol. 50, no. 3, pp. 587-601, 2018.

[18] M. Riaz and S. T. Tehrim, "Certain properties of bipolar fuzzy soft topology via Q-neighborhood," Journal of Mathematics, vol. 51, no. 3, pp. 113-131, 2019.

[19] S. Roy and T. K. Samanta, "A note on fuzzy soft topological spaces," Annals of Fuzzy Mathematics and Informatics, vol. 3, no. 2, pp. 305-311, 2012.

[20] S. Roy and T. K. Samanta, "An introduction to open and closed sets on fuzzy soft topological spaces," Annals of Fuzzy Mathematics and Informatics, vol. 6, no. 2, pp. 425-431, 2013. 